\title{
Corrosion Inhibition of Aluminum by Psidium Guajava Seeds in HCl Solution
}

\author{
Y. C. Sharma ${ }^{a, *}$ and S. Sharma ${ }^{b}$ \\ ${ }^{a}$ Faculty of Basic and Applied Sciences \& Dean, Research \& Development at Vivekananda \\ Global University, Jaipur (Rajasthan) 303 012, India- \\ ${ }^{b}$ Department of Chemistry, University of Rajasthan, Jaipur (Rajasthan), 302 015, India
}

Received April 30, 2016; accepted October 6, 2016

\begin{abstract}
The corrosion inhibition and adsorption of ethanol extracts of Psidium guajava seeds (EEPgS), for aluminum in $0.5 \mathrm{~N} \mathrm{HCl}$ solutions, were investigated using conventional weight loss, FTIR spectroscopy and SEM analysis techniques. The results showed that $\mathrm{EEPgS}$ performed well as inhibitor for the corrosion of aluminum in hydrochloric acid media. FTIR results showed that the inhibition mechanism was by adsorption process, through the functional groups present in the extract. Inhibition efficiency increased with increasing concentration of the plant extract, but decreased with the temperature rise. The weight loss data were fitted into a number of isotherms, though Langmuir model was found to be the best fit. The SEM photographs confirmed the protection offered by the extract on the surface of the metal.
\end{abstract}

Keywords: Psidium guajava; aluminum; corrosion; inhibitor; adsorption.

\section{Introduction}

Industrial processes such as prickling and acid cleaning often involve contact between a metal and aggressive solution, consequently, the metal is prone to corrosion. In order to reduce the menace due to corrosion of industrial installations, several steps have been adopted. However, one of the best options available for protecting metals against corrosion involves the use of corrosion inhibitors [1-2]. The majority of well-known inhibitors are organic compounds containing hetero atoms, such as $\mathrm{O}, \mathrm{N}, \mathrm{S}$, and multiple bonds, which allow an adsorption on the metal surface [3]. These compounds adsorb on metal surface and block the active surface sites to reduce the corrosion rate. Adsorption may take place by four different mechanisms through organic molecules at the metal/solution interface: (a) electrostatic attraction between the charged molecules and the charged metal, (b) interaction of uncharged electron pairs in

\footnotetext{
* Corresponding author. E-mail address: yogeshchandra.sharma@gmail.com, yc.sharma.vit@gmail.com
} 
the molecule with the metal, (c) interaction of $\pi$-electron with the metal, (d) combination of the first two processes, i.e., (a) and (c) b [4]. However, the efficiency of these inhibitors depends on the nature and the state of the metallic surfaces, chemical composition and structure of the inhibitor [5]. Many organic compounds have shown good anticorrosive activity, but most of them are highly toxic to both human beings and environment. According to the art of green chemistry, the research and development of nontoxic, natural and environmentally friendly inhibitors are carried out in a continuous mode, to control the corrosion phenomenon of aluminum in alkaline /acidic media. Natural products can be considered as a good source for this purpose. Extracts of naturally occurring products contain a mixture of compounds, are easily available and biodegradable in nature. Plant products are organic in nature, and some of the constituents, including tannins, organic and amino acids, alkaloids, and pigments, are known to exhibit an inhibiting action. Moreover, they can be extracted by a simple low cost procedure [6-10].

The present work describes a study of the effectiveness of ethanolic extract of Psidium guajava seeds (EEPgS) on acid corrosion of aluminum alloy (AA6063), using chemical and electrochemical methods. In this work, the synergistic improvement of the inhibition efficiency of $\mathrm{EEPgS}$, due to the presence of some ion additives, has also been investigated. The adsorption behavior of EEPgS on the surface of aluminum alloy (AA6063) was justified by FTIR spectroscopy. The protective layer formed on the metal surface was also inspected by optical and scanning electron microscopy. The inhibitory action of EEPgS was explained, and various adsorption isotherms were tested to fit in to the results. On the basis of the results, it is suggested that $\mathrm{EEPgS}$ can be used as green inhibitor for the acid corrosion of aluminum alloy (AA6063).

Scientific classification of the Guava (Psidium guajava) is as it follows:

$\begin{array}{lll}\text { Kingdom } & - & \text { Plante } \\ \text { Division } & - & \text { Mangoliophyta } \\ \text { Class } & - & \text { Mangoliophyta } \\ \text { Order } & - & \text { Myrtales } \\ \text { Family } & - & \text { Myrtaceae } \\ \text { Genus } & - & \text { Psidium } \text { L. } \\ \text { Species } & - & \text { Psidium guajava } \text { L. } \\ \text { Bionomial name } & - & \text { Psidium guajava } \text { L (apple guava) }\end{array}$

Psidium guajava, known as guava, belongs to the Myrtaceae family. Literature survey reveals that Psidium guajava seeds, which have 6 to $12 \%$ fruit weight, are found to be rich in constituents containing proteins (15\%), starch (13\%), oils (14\%), tenphenolic and flavonoid compounds, flavonolglycoside, quercetin 3 O $\beta$ D (2"Ogalloyglu-coside) 4' O vinylpropionate, amino acids like threonine, tyrosine, methionine, phenylanine, tryptophan, etc. The fat contains $11.8 \%$ saturated fatty acids and $87.3 \%$ unsaturated fatty acids, $0.1 \%$ myristic, $6.6 \%$ palmatic, $4.6 \%$ steric, $10.8 \%$ oleic, $76.4 \%$ lineolic, $0.3 \%$ arachidonic acid and $0.1 \%$ linolonic acid [11-15]. The major constituents in Psidium guajava contain 
nitrogen and oxygen compounds, and some investigations showed their adsorptive property over metal surface and anticorrosive behavior [16-20]. The structure of some chemical constituents is as it follows:<smiles>CC(C=CCO)=CC=CC(C)=CC1=C(O)CCCC1(C)C</smiles>

Quercetin<smiles>O=c1c(O)c(-c2ccc(O)cc2)oc2cc(O)cc(O)c12</smiles>

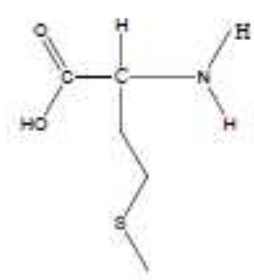

Methionine

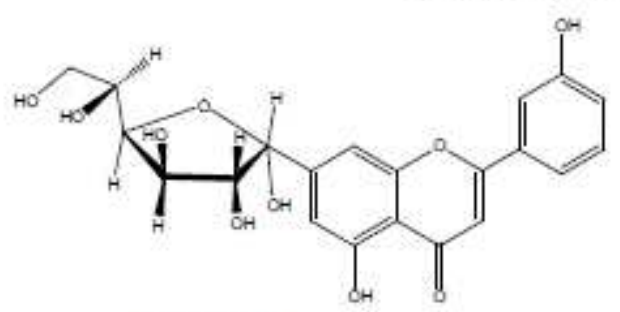

Glucoside

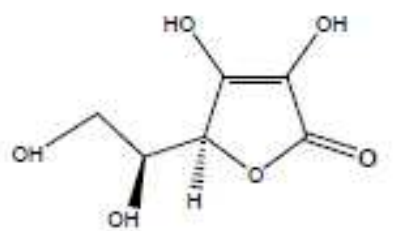

Ascorbic acid

\section{Materials and methods}

To determine the inhibitive properties of $\mathrm{EEPgS}$ on acid corrosion of aluminum alloy (AA6063), weight loss and electrochemical methods were employed. All the aggressive solutions $(0.5 \mathrm{~N} \mathrm{HCl})$ used were made of AR grade reagent and deionized water. All sets of experiments were performed in triplicate to confirm the reproducibility of the results. Preparation of test solutions, cleaning of aluminum coupons and preparation of ethanolic extract of $P g \mathrm{~S}$ were done as per standard practices [21-23].

\section{Preparation of aluminum coupons}

The industrially used aluminum of $98.99 \%$ purity (AA6063) has been used as test coupon. For weight loss experiments, mechanically press cut rectangular aluminum coupons $(3 \mathrm{~cm} \times 2 \mathrm{~cm} \times 0.16 \mathrm{~cm})$, with a hole of about $0.12 \mathrm{~mm}$ diameter near the upper edge, were used. They were carefully examined to check out for rough edges, and the surface treatment of the coupons was carried out by using various grades of emery paper (110 to 410), until the surface appeared free from scratches and other apparent defects. These were degreased in acetone and finally washed with bi-distilled water and then dried in an oven. Thereafter, these specimens were kept in desiccators, until they cooled to room temperature, and then they were accurately weighed using a digital balance. Afterwards, they were subjected to heating, cooling and weighing, until a constant weight was obtained, 
and then kept in desiccators. The surface treatment was carried out immediately before each experiment of corrosion.

\section{Preparation of ethanol extract of Psidium guajava seeds (EEPgS)}

The stock solutions of the Psidium guajava seeds were prepared by soaking the weighed amount of room temperature dried and finely powdered seeds of Psidium guajava in a properly corked container containing distilled ethanol. The mass of $P g S$ was accessed to be $60.3 \mathrm{~g} / \mathrm{L}$ of plant compound. On completion of soaking period, the ethanolic solutions were refluxed for $24 \mathrm{~h}$, to concentrate the inhibiting chemicals, and then filtered to remove any suspended impurities. This stock solution of the extract was stored in a clean corked bottle. From this dried liquid, Psidium guajava seeds extract was used as inhibitor.

\section{Preparation of test solutions}

The aggressive solutions were measured to $100 \mathrm{~mL}$ in seven separate beakers, labeled as S0, S1, S2, S3, S4, S5 and S6. EEPgS were added in order of increasing concentration, as to have $0.1809,0.3015,0.4221,0.7236$ and 1.2066 g/L, respectively, in S1, S2, S3, S4, S5 and S6 beaker solution, while no extract was added to the S0, i.e., the first beaker solution. All the beakers were kept unstirred and covered with teflon tape throughout the experiment.

\section{Fourier-transform infrared (FTIR) spectroscopy}

FTIR analysis was carried out to determine the functional groups present in the $\mathrm{EEPgS}$ and in the corrosion products from the aluminum corrosion set ups containing the EEPgS extract. The spectra were recorded with an $8400 \mathrm{~s}$ Shimadzu, Japan spectrometer by scanning the samples through a wave number range of 400 to $4000 \mathrm{~cm}^{-1}$. The samples were prepared for the analysis using $\mathrm{KBr}$.

\section{Scanning electron microscopy}

The surface morphology of the aluminum, before and after immersion in the corrosion media, were examined using SEM machine model- Quanta 3D FEG.

\section{Results and discussion \\ FTIR analysis of the extract and corrosion product}

Figs. 1 and 2 show the FTIR spectra of the EEPgS and the corrosion product, and Table 1 presents the data. It was found that the N-H stretching was at $2350 \mathrm{~cm}^{-1}$, which was shifted to $2360 \mathrm{~cm}^{-1}$, cyclic five member aldehyde and ketone were at $1750 \mathrm{~cm}^{-1}$, which shifted to $1900 \mathrm{~cm}^{-1}$, and $\mathrm{C}=\mathrm{C}$ benzene ring at $1660 \mathrm{~cm}^{-1}$ was shifted to $1650 \mathrm{~cm}^{-1}$. Shifts were also observed in other peaks. The shifts in the spectra indicate that the interaction between the extracts and aluminum occurred through the functional groups present in EEPgS. Moreover, it can be affirmed that the functional groups have coordinated with the $\mathrm{Al}^{3+}$ formed on the metal surface, resulting in the formation of $\mathrm{Al}^{3+}$ extract complex on the metal surface, which promotes the inhibition of the aluminum sample surface [26-27]. 


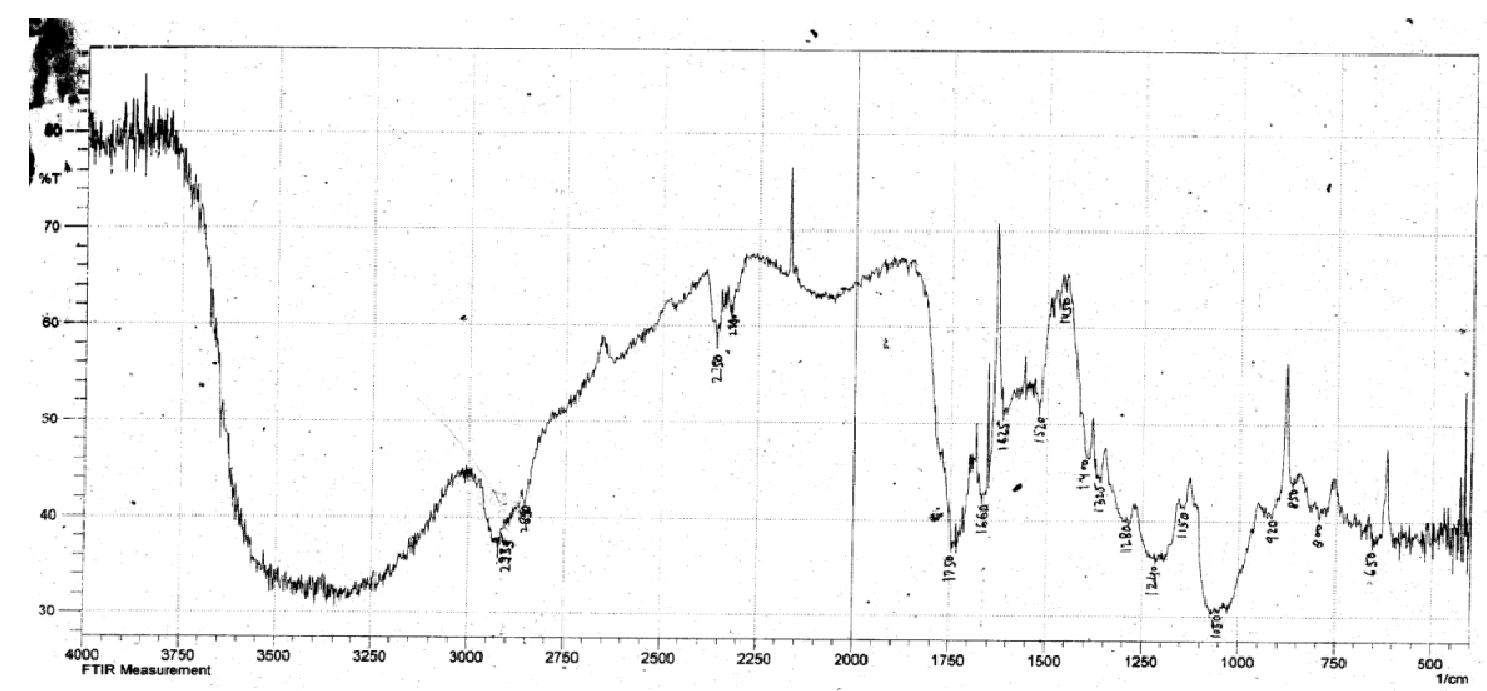

Figure 1. FTIR spectrum of ethanol extract of Psidium guajava seeds (EEPgS).

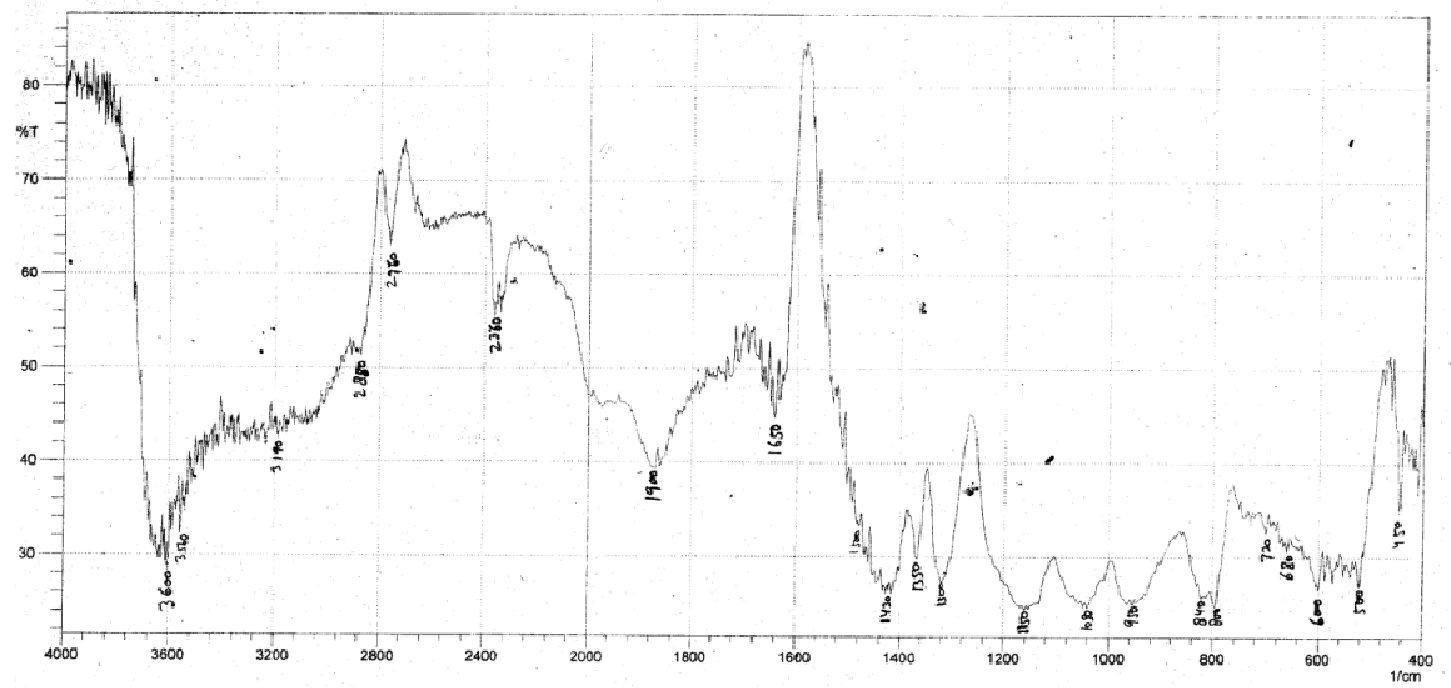

Figure 2. FT-IR spectra of adsorbed film of EEPgS over aluminum alloy (AA6063) exposed in $0.5 \mathrm{~N} \mathrm{HCl}$ containing highest concentration of inhibitor.

\section{Weight loss measurements}

The effect of the inhibitor on the acid corrosion of aluminum was studied using the weight loss technique at room temperature, at various exposure time periods. The variation of weight loss in the absence and presence of the various concentrations of EEPgS has been plotted in Fig. 3. It can be observed that weight loss increased with increase in exposure time. It has been also observed that addition of EEPgS resulted in a significant reduction in the material loss, in comparison with the blank solution. The reduction in the mass loss in the presence of the extract has been attributed to the adsorption of the constituents of EEPgS (Table 1). The adsorption of such compounds on the metal surface creates a barrier for charge and mass transfer, leading to a decrease in the interaction between the metal and the corrosion solution. The presence of this barrier film inhibits the metal surface corrosion [28]. 
Table 1. Peaks of IR adsorbed on aluminum alloy (AA6063) and EEPgS.

\begin{tabular}{|c|c|c|c|}
\hline \multicolumn{2}{|c|}{ Pure extract of EEPgS } & Extract adsorbed on alloy AA6063 \\
\hline $\begin{array}{c}\text { Frency } \mathbf{~}) \\
2950\end{array}$ & Assigned peak & $\begin{array}{c}\text { Frequency } \\
\left(\mathbf{c m}^{-1}\right)\end{array}$ & Assigned peak \\
\hline 2850 & $\begin{array}{c}\text { C-H stretching } \\
\text { C-H stretching }\end{array}$ & 2880 & $\begin{array}{c}\text { Aliphatic and } \\
\text { aromatic C-H } \\
\text { stretching }\end{array}$ \\
\hline 2350 & N-H stretching & 2360 & N-H stretching \\
\hline 1750 & $\begin{array}{c}\text { Cyclic five membered } \\
\text { aldehyde and ketone }\end{array}$ & 1900 & $\begin{array}{c}\text { Cyclic five } \\
\text { membered aldehyde } \\
\text { and ketone }\end{array}$ \\
\hline 1660 & $\begin{array}{c}\text { Aromatic C=C with } \\
\text { benzene rings }\end{array}$ & 1650 & N-H def. \\
\hline 1450 & Aromatic C=C & 1430 & Aromatic C=C \\
\hline 1325 & $\begin{array}{c}\text { C-O stretching, primary } \\
\text { alcohol }\end{array}$ & 1300 & primary alcohol \\
\hline 1280 & $\begin{array}{c}\text { Aliphatic, aromatic C-H } \\
\text { stretching }\end{array}$ & & \\
\hline 1150 & $\begin{array}{c}\text { C-O stretching in aromatic } \\
\text { ether }\end{array}$ & & \\
\hline 1020 to 1200 & tertiary alcohol & & \\
\hline 920 & aliphatic amine & & \\
\hline 800 to 850 & mono substituted alkenes & & \\
\hline
\end{tabular}

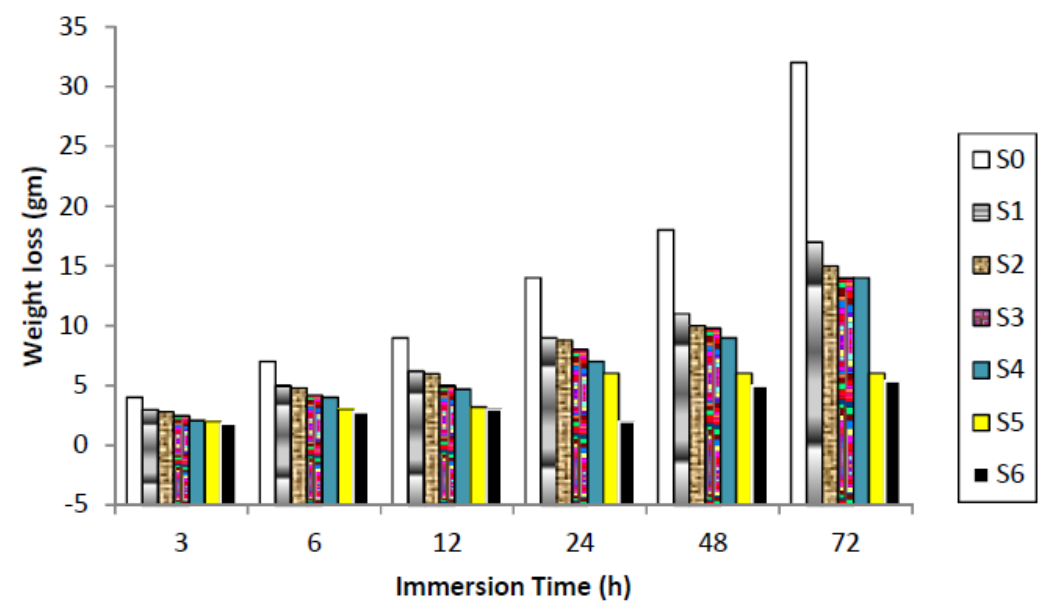

Figure 3. Weight loss (mg) - immersion time (h) graphs for the corrosion of aluminum in $0.5 \mathrm{~N} \mathrm{HCl}$, in the absence and in the presence of different concentrations (S0-S6) of $\mathrm{EEPgS}$ at room temperature $(303 \pm 1 \mathrm{~K})$.

Fig. 4 shows that, as the inhibitor concentration is increased, the rate of corrosion decreases. The corrosion rate, with respect to the immersion time period at lower concentrations, sharply decreases up to $48 \mathrm{~h}$; after that, with an increased immersion time period of $72 \mathrm{~h}$, a slightly increase in corrosion rate was observed. But, at higher concentrations, the corrosion rate decreased with an increase in the immersion period. The minimum corrosion rate was found to be $0.1509 \mathrm{mmy}^{-1}$ at 
$72 \mathrm{~h}$, for the highest inhibitor's concentration, $1.206 \mathrm{~g} / \mathrm{L}$. This clearly indicates that, at a higher immersion time period, the corrosion rate was found to be independent of the EEPgS' concentration.

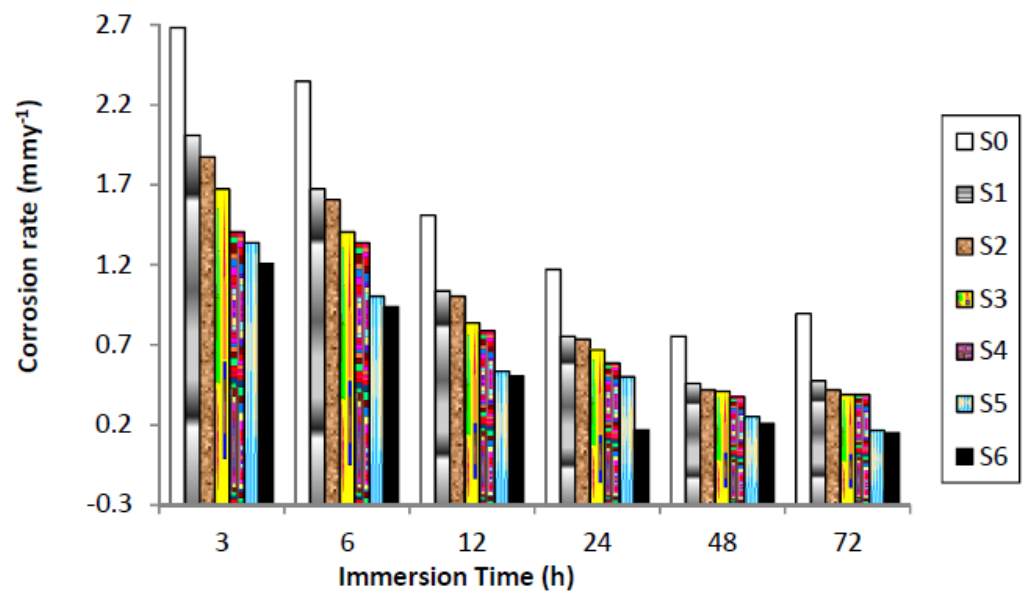

Figure 4. Corrosion rate - immersion time graphs for the corrosion of aluminum in 0.5 $\mathrm{N} \mathrm{HCl}$, in the absence and in the presence of different concentrations (S0-S6) of EEPgS at room temperature $(303 \pm 1 \mathrm{~K})$.

The dissolution of $\mathrm{Al}^{3+}$ in the electrolyte was examined by using Atomic Absorption Spectrometer (AAS) analysis. Concentration of $\mathrm{Al}^{3+}$ ions, as a function of the extract concentration, has been shown in Fig. 5. This plot confirms the results obtained from the weight loss measurements.

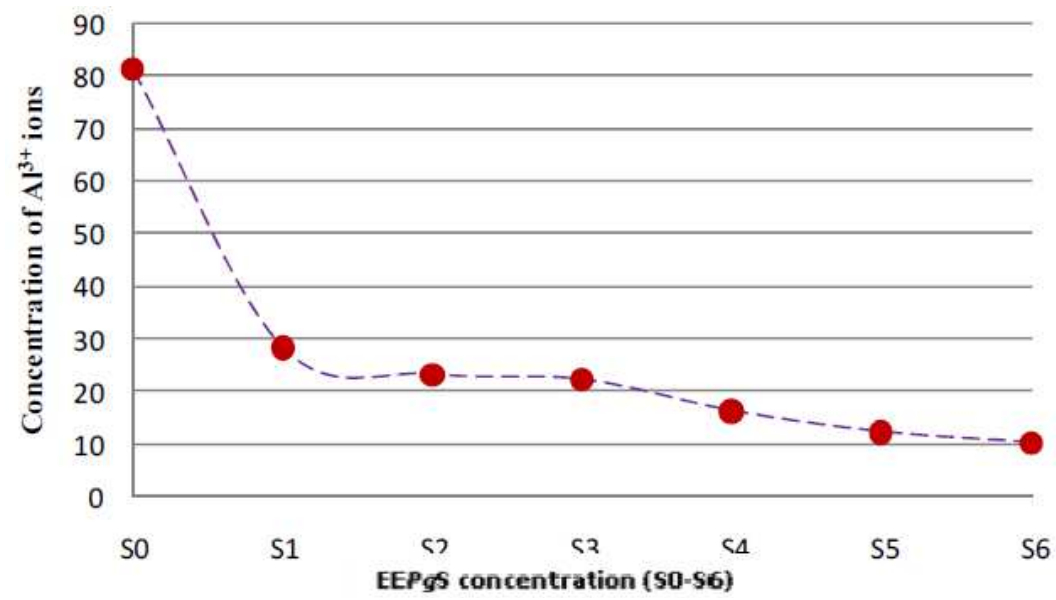

Figure 5. Plot of concentration of $\mathrm{Al}^{3+}$ ions in $0.5 \mathrm{~N} \mathrm{HCl}$, in the presence and absence of the $\mathrm{EEPgS}$.

The plot shows a steady decrease in the concentration of $\mathrm{Al}^{3+}$ ions in the electrolyte as more extract was added, which indicates the adsorption of the extract on the metal surface. Adsorption of the inhibitor species on the metal surface reduced the oxidation of aluminum atom from $\mathrm{Al}$ to $\mathrm{Al}^{3+}$.

\section{Effect of temperature on inhibition efficiency and corrosion rate}

The effect of temperature variation is very complex, because many changes occur on the metal surface, like desorption of the inhibitor from the metal surface, rapid 
etching and, moreover, the inhibitor may undergo decomposition at high temperatures.

The values of inhibition efficiency (IE\%) obtained from weight loss method, at different concentrations and at different temperatures for $24 \mathrm{~h}$, have been reported in Table 2. It is seen from the data and Fig. 6 that IE\% reaches a maximum value of $85.71 \%$ with the highest concentration of inhibitor (1.206 $\mathrm{g} / \mathrm{L})$, but it decreases with an increase in temperature. Decrease in inhibition efficiency with increase in temperature is suggestive of a physical adsorption mechanism and may be attributed to increases in the solubility of the protective film, and to any reaction of the product precipitated on the surface of aluminum alloy (AA6063) that may, otherwise, inhibit the corrosion process. It may further be attributed to a possible shift of the adsorption-desorption equilibrium towards desorption of the adsorbed inhibitor, due to increased solution agitation. At higher temperature, there is highest IE\% shifts towards lower concentration, which means that many constituents are more effective, the nature of solution changes, and a greater number of molecules shifts from the surface, due to thermal agitation. Quercetin is the flavonoid compound present in the EEPgS, with conjugated system, and contains hetero atoms and carbonyl groups which are electron rich. At higher temperature, many monomer units of quercetin combine with each other, forming a polymer which can serve as a good adsorption site onto the metal surface at higher temperature, thereby inhibiting corrosion [29].

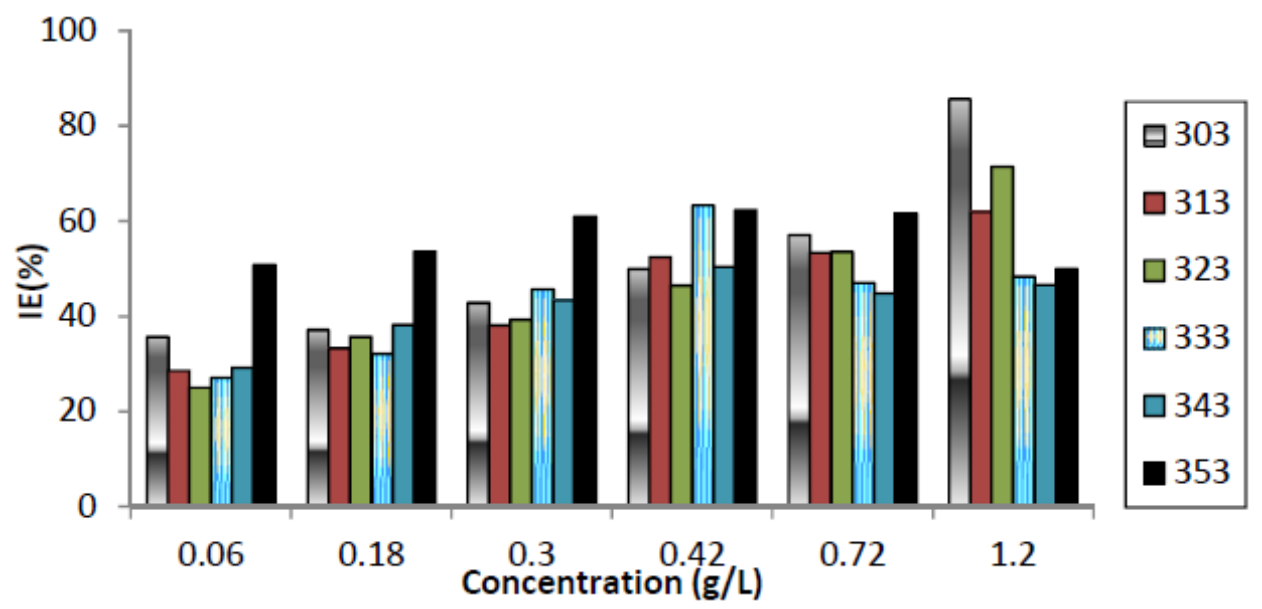

Figure 6. Inhibition efficiency (IE \%) against various concentrations of EEPgS at various temperatures (303-353 K) studied, using weight loss method.

Table 2. Corrosion rates of aluminum in $0.5 \mathrm{~N} \mathrm{HCl}$, in the absence and presence of $\mathrm{EEPgS}$.

\begin{tabular}{|c|c|c|c|c|c|c|}
\hline EEPgS concentration & $\mathbf{3 0 3} \mathbf{~ K}$ & $\mathbf{3 1 3} \mathbf{K}$ & $\mathbf{3 2 3} \mathbf{~ K}$ & $\mathbf{3 3 3} \mathbf{~ K}$ & $\mathbf{3 4 3} \mathbf{~ K}$ & $\mathbf{3 5 3} \mathbf{~ K}$ \\
\hline S0 & 1.1735 & 1.7602 & 2.3470 & 18.5243 & 17.7699 & 32.6899 \\
\hline S1 $(0.0603)$ & 0.7544 & 1.2573 & 1.7602 & 13.4950 & 12.5730 & 16.0935 \\
\hline S2 $(0.1809)$ & 0.7376 & 1.1735 & 1.5088 & 12.5730 & 10.9804 & 15.1715 \\
\hline S3 $(0.3015)$ & 0.6706 & 1.0897 & 1.4249 & 10.0584 & 10.0584 & 12.7407 \\
\hline S4 $(0.4221)$ & 0.5867 & 0.8382 & 1.2573 & 6.7894 & 8.8011 & 12.3216 \\
\hline S5 $(0.7236)$ & 0.5029 & 0.8214 & 1.0897 & 9.8070 & 9.8070 & 12.5730 \\
\hline S6 (1.2060) & 0.1676 & 0.6706 & 0.6706 & 9.5555 & 9.4717 & 16.3449 \\
\hline
\end{tabular}


Table 2 shows the values of corrosion rate at different temperatures and concentrations of the extract, for an immersion time of $24 \mathrm{~h}$. From Table 2, it is seen that the corrosion rate increases with an increase in temperature. This is as expected because, as the temperature increases, the average kinetic energy of the reacting molecules increases, thereby speeding up the reaction rate. It is also seen that the corrosion rate values decrease with an increase in the concentration of $\mathrm{EEPgS}$.

\section{Kinetic treatment of weight loss results}

The corrosion reaction of aluminum against the concentration of the studied inhibitor $(\mathrm{EEPgS})$ shows the kinetic relationship:

$$
\log \rho_{\text {corr }}=\log \mathrm{k}+\mathrm{B} \log \mathrm{C}_{\mathrm{inh}}
$$

where $\mathrm{k}$ is the rate constant and is equal to $\rho_{\text {corr }}$ at unity inhibitor concentration, $\mathrm{B}$ is the reaction constant which, in the present case, is the measure for the inhibitor effectiveness, and $\mathrm{C}_{\mathrm{inh}}$ is the concentration of $\mathrm{EEPgS}$. Fig. 7 represents the curves of $\log \rho_{\text {corr versus }} \log \mathrm{C}_{\mathrm{inh}}$ at various temperatures, for an immersion time period of $24 \mathrm{~h}$. The straight lines show that the kinetic parameters ( $\mathrm{k}$ and B) could be calculated by equation 1 . The data obtained from the plots are tabulated in Table 3.

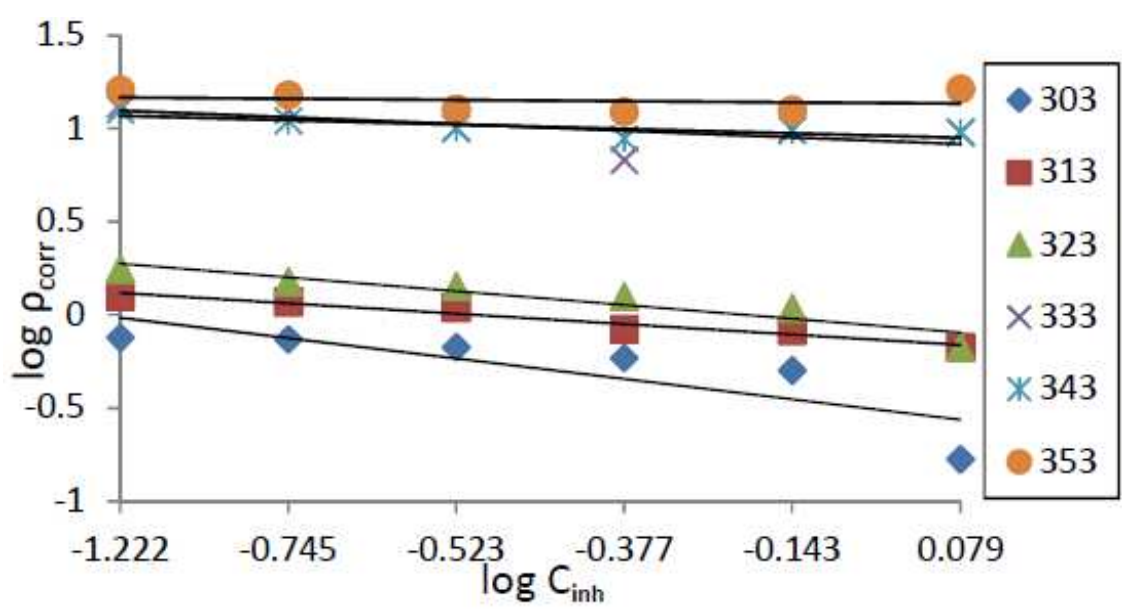

Figure 7. Curve fitting of corrosion rate against inhibitor concentration for aluminum in $0.5 \mathrm{~N} \mathrm{HCl}$, in the presence and absence of various concentrations of EEPgS at different temperatures, for $24 \mathrm{~h}$.

Table 3. Kinetic parameters for the corrosion of aluminum alloy (AA6063) in $0.5 \mathrm{~N}$ $\mathrm{HCl}$, for various concentrations of $\mathrm{EEPgS}$ at different temperatures, for $24 \mathrm{~h}$.

\begin{tabular}{|c|c|c|c|}
\hline \multirow{2}{*}{$\begin{array}{c}\text { Temperature } \\
(\mathbf{K})\end{array}$} & \multicolumn{3}{|c|}{$\mathbf{2 4 h}$} \\
\cline { 2 - 4 } & $\mathbf{B}$ & $\mathbf{k}$ & $\mathbf{R}^{\mathbf{2}}$ \\
\hline 303 & -0.109 & 4.954 & 0.682 \\
\hline 313 & -0.055 & 5.942 & 0.953 \\
\hline 323 & -0.073 & 8.892 & 0.872 \\
\hline 333 & -0.035 & 53.951 & 0.399 \\
\hline 343 & -0.023 & 49.310 & 0.652 \\
\hline 353 & -0.006 & 59.421 & 0.044 \\
\hline
\end{tabular}


From data in Table 3 it is seen that the reaction constant B has negative sign, which means the corrosion reaction is inversely dependent on the inhibitor's concentration.

It can also be seen that there is a decrease in the negative value of B with an increase in temperature. The $\mathrm{k}$ value increases with rising temperature. At each temperature, a linear variation was observed in the presence of EEPgS, thus confirming a first order kinetics [30].

\section{Kinetic parameters of energy of activation}

The temperature study on the kinetic process of corrosion in corrosive medium $(0.5 \mathrm{~N} \mathrm{HCl})$, in the presence of adsorbed inhibitor, provides more information about the nature of adsorption to evaluate the activation process. The corrosion reaction of aluminum alloy in acidic medium depends on temperature. The log of corrosion rate is a linear function of temperature (Arrhenius equation):

The relationship of corrosion rate $(\rho)$ of aluminum in acidic medium and temperature $(\mathrm{T})$ is given by Arrhenius equation:

$$
\ln p_{\text {corr }}=\ln A-\frac{B_{\mathrm{g}}}{\mathrm{RT}}
$$

where $\rho_{\text {corr }}$ is the corrosion rate, $E_{a}$ is the apparent activation energy, $\mathrm{R}$ is the molar gas constant $\left(8.314 \mathrm{~J} \mathrm{~K}^{-1} \mathrm{~mol}^{-1}\right), \mathrm{T}$ is the absolute temperature, and $\mathrm{A}$ is the frequency parameter.

The plot of $\ln \rho_{\text {corr }}$ Vs. 1/T gave a straight line, as shown in Fig. 8, and the values of $\mathrm{E}_{\mathrm{a}}$ for aluminum alloy (AA6063) in acidic medium, in the absence and presence of various concentrations of $\mathrm{EEPgS}$, were calculated from the slopes of ln $\rho_{\text {corr }}$ vs. $1 / \mathrm{T}$, which are tabulated in Table 4.

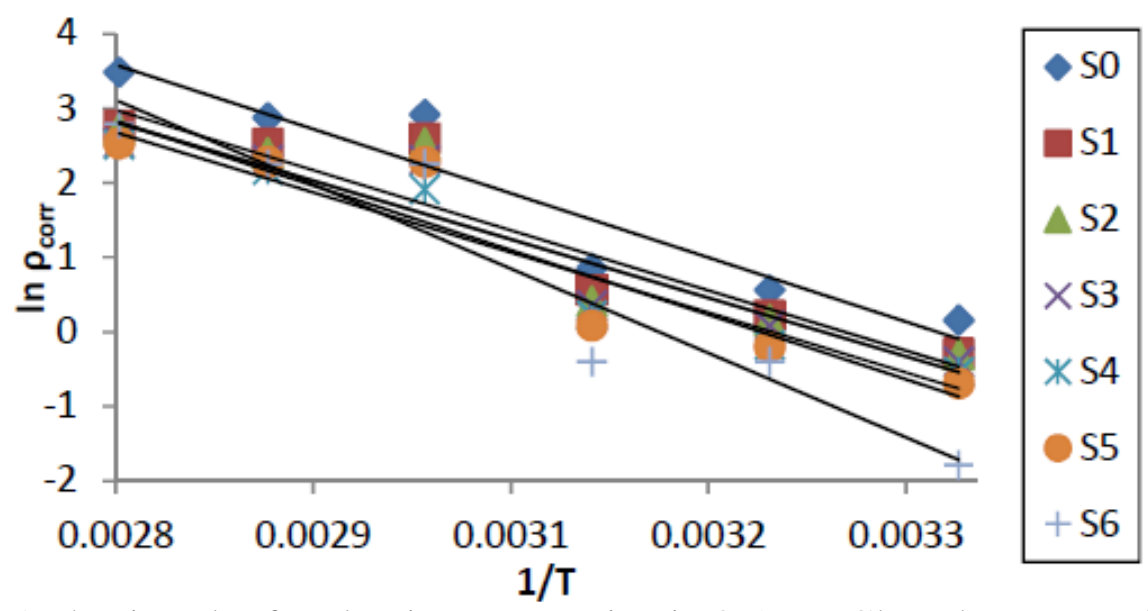

Figure 8. Arrhenius plot for aluminum corrosion in $0.5 \mathrm{~N} \mathrm{HCl}$, and extracts of $P g \mathrm{~S}$ with different concentrations at various temperatures (303-353 K).

The kinetic parameters, the enthalpy of activation $(\Delta \mathrm{H})$ and the entropy of activation $(\Delta S)$ for the corrosion of aluminum alloy (AA6063) in acidic medium were obtained by transition state equation [30]:

$$
\log \frac{P_{\text {gorr }}}{T}=\left[\log \left(\frac{R}{N h}\right)+\left(\frac{\Delta S}{2 \cdot 3 \cdot 03 R}\right)\right]-\frac{\Delta H}{2 \cdot 303 R T}
$$


where $\mathrm{h}$ is the Plank's constant, $\mathrm{N}$ is the Avogadro's number, $\Delta \mathrm{H}$ is the enthalpy of activation and $\Delta S$ is the entropy of activation. A plot of $\log \frac{\rho_{\text {sorr }}}{T}$ versus $\frac{1}{T}$ produces a straight line (Fig. 9) with a slope of $\frac{\Delta \mathrm{H}}{2,303 \mathrm{RT}}$ and an intercept of $\left[\log \left(\frac{\mathbf{R}}{\mathrm{Nh}}\right)+\left(\frac{\Delta \mathrm{S}}{2.303 \mathrm{R}}\right)\right]$, from which the values of $\Delta \mathrm{H}$ and $\Delta \mathrm{S}$ were calculated and presented in Table 4.

Table 4. Activation parameters of acid corrosion of aluminium alloy (AA6063), with and without various concentrations of $\mathrm{EEPgS}$ at different temperatures (303-353 K), for $24 \mathrm{~h}$.

\begin{tabular}{|c|l|l|l|}
\hline \multirow{2}{*}{ Solution } & \multicolumn{3}{|l|}{$\mathbf{2 4 ( \mathbf { h } )}$} \\
\cline { 2 - 4 } & $\begin{array}{c}\mathbf{E}_{\mathbf{a}} \\
(\mathbf{k J} / \mathbf{m o l})\end{array}$ & $\begin{array}{c}\Delta \mathbf{H} \\
(\mathbf{k J} / \mathbf{m o l})\end{array}$ & $\begin{array}{c}\Delta \mathbf{S} \\
(\mathbf{k J} / \mathbf{m o l})\end{array}$ \\
\hline S0 & 65.08 & -62.38 & -39.98 \\
\hline S1 & 59.47 & -59.10 & -53.52 \\
\hline S2 & 60.99 & -58.28 & -56.58 \\
\hline S3 & 59.47 & -56.75 & -62.22 \\
\hline S4 & 60.87 & -58.16 & -59.43 \\
\hline S5 & 65.53 & -62.82 & -48.40 \\
\hline S6 & 85.45 & -82.75 & 13.81 \\
\hline
\end{tabular}

In $0.5 \mathrm{~N} \mathrm{HCl}$ solution, the addition of $\mathrm{EEPgS}$ leads to an increase in the apparent activation energy to a value greater than that of the uninhibited solution, indicating physical adsorption of the extract on the metal surface. Decrease in the surface available for corrosion, due to the addition of EEPgS up to $1.24 \mathrm{~g} / \mathrm{L}$ in $0.5 \mathrm{~N} \mathrm{HCl}$, increases the activation energy from 65.08 to $85.45 \mathrm{~kJ} / \mathrm{mol}$. The results show that the addition of $\mathrm{EEPgS}$ decreases metal dissolution in $0.5 \mathrm{~N} \mathrm{HCl}$ medium. This hindrance to dissolution is due to the formation of the metal complex layer [31-32].

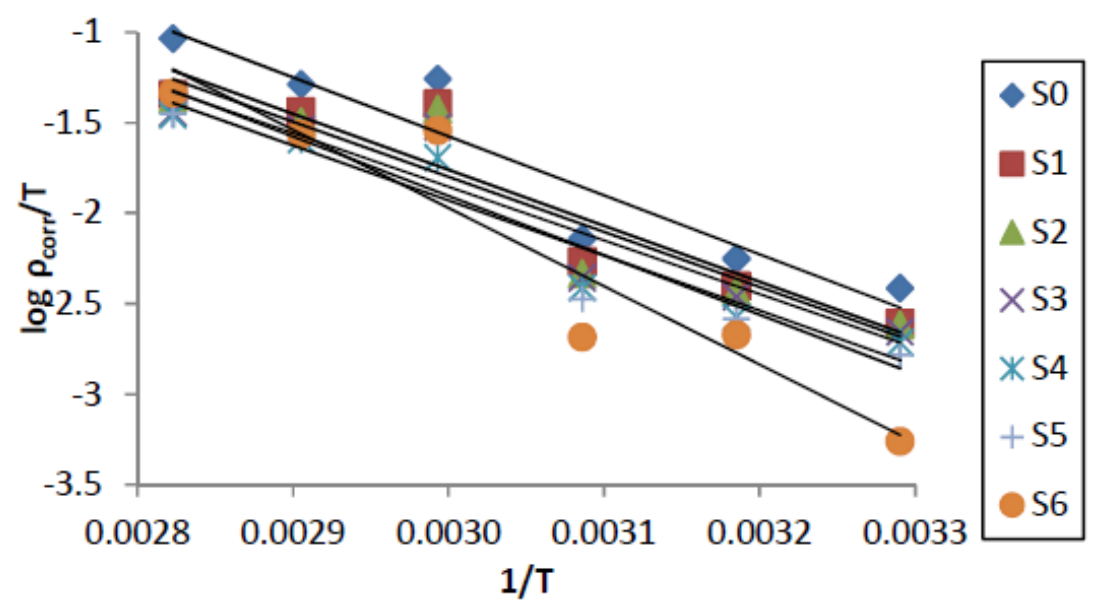

Figure 9. Transition state plots for aluminum corrosion in $0.5 \mathrm{~N} \mathrm{HCl}$, in the presence and absence of different inhibitor's concentrations.

The negative sign of enthalpy $(\Delta \mathrm{H})$ reveals that the adsorption of inhibitor molecules is an exothermic process. The shift towards positive values of entropy 
$(\Delta \mathrm{S})$ implies that the activated complex in the rate determining step represents dissociation rather than association, meaning that disordering increases on going from the reactant to the activated complex.

The results show that the enthalpy of activation values are all positive, which reflects the endothermic nature of the aluminum dissolution process. Also, the enthalpy of activation in the presence of the extracts is higher than in the blank solution. This indicates that the aluminum dissolution requires more energy in the presence of the extract than in its absence.

From the known thermodynamic relation between $\mathrm{E}_{\mathrm{a}}$ and $\Delta \mathrm{H}$ [19-25]:

$$
\mathrm{E}_{\mathrm{a}}-\Delta \mathrm{H}=\mathrm{RT}
$$

The average difference value of the $\mathrm{E}_{\mathrm{a}^{-}} \Delta \mathrm{H}$ is $2.7 \mathrm{~kJ} / \mathrm{mol}$, which is approximately equal to the average value of RT $(2.63 \mathrm{~kJ} / \mathrm{mol})$. This indicates that the corrosion reaction is a unimolecular reaction. This result shows that the inhibitor acted equally on $\mathrm{E}_{\mathrm{a}}$ and $\Delta \mathrm{H}$.

\section{Thermodynamic parameters}

Thermodynamic parameters are also determined from the experimental data. The most important thermodynamic adsorption parameters are the free energy of adsorption $\left(\Delta \mathrm{G}_{\mathrm{ads}}\right)$, the heat of adsorption $\left(\Delta \mathrm{H}_{\mathrm{ads}}\right)$ and the entropy of adsorption $\left(\Delta \mathrm{S}_{\mathrm{ads}}\right)$. The standard free energy of adsorption $\left(\Delta \mathrm{G}_{\mathrm{ads}}\right)$, which characterizes the interaction of adsorption molecules and metal surface, alongwith other parameters, was evaluated using the following equation:

$$
\Delta \mathrm{G}_{\mathrm{ads}}=\mathrm{RT} \ln \left(\mathrm{K}_{\mathrm{ad}} \mathrm{x} 55.5\right)
$$

where 55.5 is the water concentration of solution $(\mathrm{mL} / \mathrm{L})$.

Table 5. Thermodynamic parameters of the acid corrosion of aluminum alloy (AA6063) with and without various concentrations of EEPgS at different temperatures

\begin{tabular}{|c|c|c|c|c|c|c|c|c|}
\hline \multirow[b]{2}{*}{ Solution } & \multicolumn{6}{|c|}{ (-) $\Delta \mathbf{G}_{\text {ads }}=\mathbf{k J} / \mathbf{m o l}$} & \multirow{2}{*}{$\begin{array}{c}\Delta \mathbf{H}_{\text {ads }} \\
(\mathbf{k J} / \mathbf{m o l})\end{array}$} & \multirow{2}{*}{$\begin{array}{c}\Delta \mathbf{S}_{\text {ads }} \\
(\mathbf{k J} / \mathbf{m o l})\end{array}$} \\
\hline & $303 \mathrm{~K}$ & $313 \mathrm{~K}$ & $323 \mathrm{~K}$ & $333 \mathrm{~K}$ & $343 \mathrm{~K}$ & $353 \mathrm{~K}$ & & \\
\hline S1 & 15.72 & 15.39 & 15.93 & 16.17 & 16.96 & 20.13 & -13.87 & -78.0 \\
\hline S2 & 13.11 & 13.11 & 13.81 & 13.79 & 14.97 & 17.24 & -11.72 & -74.0 \\
\hline S3 & 12.42 & 12.32 & 12.85 & 13.97 & 14.13 & 16.63 & -10.96 & -78.0 \\
\hline S4 & 12.30 & 12.95 & 12.73 & 15.03 & 13.98 & 15.81 & -11.51 & -65.0 \\
\hline S5 & 11.67 & 11.65 & 12.05 & 11.70 & 11.79 & 14.33 & -10.93 & -35.0 \\
\hline S6 & 14.17 & 11.24 & 12.75 & 10.44 & 10.55 & 11.25 & -13.63 & 54.0 \\
\hline
\end{tabular}
(303-353 K), at the immersion time of $24 \mathrm{~h}$.

The thermodynamic parameters $\Delta \mathrm{H}_{\mathrm{ads}}$ and $\Delta \mathrm{S}_{\mathrm{ads}}$ for the adsorption of EEPgS on aluminum were calculated from the following equation:

$$
\Delta \mathrm{G}_{\mathrm{ads}}=\Delta \mathrm{H}_{\mathrm{ads}}-\mathrm{T} \Delta \mathrm{S}_{\mathrm{ads}}
$$

where $\Delta \mathrm{H}_{\mathrm{ads}}$ and $\Delta \mathrm{S}$ ads are the variations of enthalpy and entropy of the adsorption process. The dependence of $\Delta \mathrm{G}_{\mathrm{ads}}$ on $\mathrm{T}$ indicates an appropriate 
relationship between thermodynamic parameters. The calculated values are given in Table 5.

The negative values of $\Delta \mathrm{G}_{\mathrm{ads}}$ ensure the spontaneity of adsorption process and stability of the adsorbed layer on the aluminum surface. Generally, the values of $\Delta \mathrm{G}_{\text {ads }}$ around $-20 \mathrm{~kJ} / \mathrm{mol}$ or lower are consistent with physisorption, while those around $-40 \mathrm{~kJ} / \mathrm{mol}$ or higher involve chemisorptions. In the present case of study, the $\Delta \mathrm{G}_{\mathrm{ads}}$ values were found to be lower than $-20 \mathrm{~kJ} / \mathrm{mol}$, which indicates physical adsorption of the molecule on the aluminum alloy (AA6063) surface. The negative sign of $\Delta \mathrm{H}$ reveals that the adsorption of the inhibitor molecules is an exothermic process. The shift towards a positive value of entropy $(\Delta S)$ implies that the spontaneous adsorption and activated complex formation in the rate determining step represents dissociation rather than association, which clearly indicates that the disordering increases on going from the reactant to the activated complex.

\section{Adsorption isotherm}

Adsorption isotherms are very important in determining the mechanism of corrosion reaction. The most frequently used isotherms are Langmuir, Frumkin, Hill de-Boer, Parsons, Temkin, Flory-Huggin, Freundlich, Bhar-Flory-Huggin, Kinetic /thermodynamic model of El-Wady et al. and Bockris-Swinkles. All these isotherms are of the general form:

$$
\mathrm{F}(\Theta, \chi) \exp ^{(-2 \mathrm{a} \theta)}=\mathrm{K}_{\mathrm{ads}} \mathrm{C}
$$

where $F(\Theta, \chi)$ is the configuration factor which depends upon the physical model and the assumption underlying the derivation of the isotherm, $\theta$ is the surface coverage, $\mathrm{C}$ is the inhibitor concentration in the electrolyte, $\chi$ is the size factor ratio, $\mathrm{a}$ is the molecular interaction parameter and $\mathrm{K}_{\mathrm{ads}}$ is the equilibrium constant of the adsorption process. The degree of surface coverage $\theta$ for various concentrations of the inhibitor has been calculated by weight loss technique.

In this study, Langmuir adsorption isotherm was found to be suitable for the experimental findings, and has been used to describe the adsorption characteristic of this inhibitor. Langmuir isotherm [33] was tested for its fit to the experimental data. Langmuir isotherm is given by

$$
\mathrm{C} / \theta=1 / \mathrm{K}_{\mathrm{ads}}+\mathrm{C}
$$

where $\theta$ is the degree of surface coverage, $\mathrm{C}$ is the molar inhibitor concentration in the bulk solution and $\mathrm{K}_{\mathrm{ads}}$ is the equilibrium constant of the process of adsorption. The plot of $\mathrm{C} / \theta$ versus $\mathrm{C}$ was straight line graphs (Fig. 10), which proves that Langmuir adsorption isotherm is obeyed for each immersion time over the range of concentration studied. The correlation coefficients and slopes obtained from Langmuir isotherm plots are shown in Table 6. 


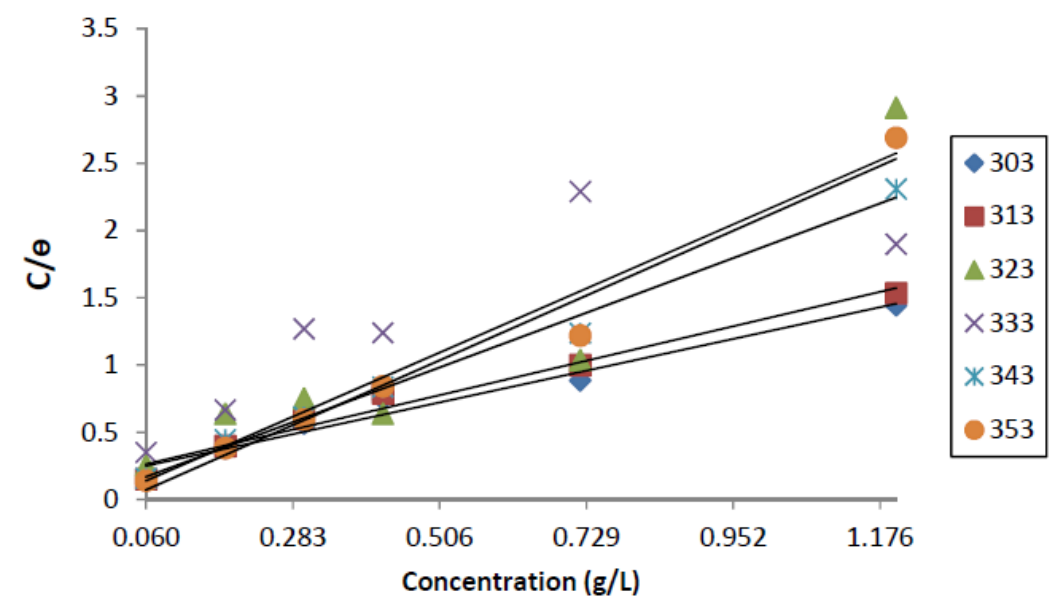

Figure 10. Langmuir adsorption isotherm plots for the adsorption of $0.5 \mathrm{~N} \mathrm{HCl}$ and different concentrations of EEPgS for various temperatures (303-353 K).

The degree of linearity of Langmuir adsorption isotherm was measured by the value of $R^{2}$ in the range $\left(0.991 \geq r^{2} \geq 0.691\right)$, which is nearly equal to one, and the slope almost reaches unity at room temperature. This indicates that the assumption and the deductions are correct. Monolayers of the inhibitor species must have been attached to the aluminum alloy surface without lateral interaction between adsorbed species. But, at higher temperatures, the deviation of the slopes from unity (for the ideal Langmuir adsorption isotherm) can be attributed to the molecular interaction among the adsorbed inhibitor species.

Table 6. Langmuir adsorption isotherm parameters at different temperatures.

\begin{tabular}{|c|c|c|}
\hline Temperature (K) & Slope & Correlation coefficient $\left(\mathbf{R}^{\mathbf{2}}\right)$ \\
\hline 303 & 1.05 & 0.968 \\
\hline 313 & 1.1 & 0.974 \\
\hline 323 & 2.1 & 0.882 \\
\hline 333 & 1.4 & 0.691 \\
\hline 343 & 1.8 & 0.991 \\
\hline 353 & 2.1 & 0.973 \\
\hline
\end{tabular}

\section{Electrochemical measurements}

Fig. 11 represents the potentiodynamic polarization curves for aluminum, in the absence and presence of different concentrations on EEPgS at $303 \mathrm{~K}$. The polarization curves show Tafel behavior.

The numerical values of the potentiodynamic polarization parameters, i.e., corrosion current density $\left(\mathrm{i}_{\text {corr }}\right)$, corrosion potential $\left(\mathrm{E}_{\mathrm{corr}}\right)$, Tafel slopes $\left(\beta_{\mathrm{a}}\right.$ and $\beta_{c}$ ), degree of surface coverage $(\theta)$, and inhibition efficiency (IE\%), with the concentration of the inhibitor are given in Table 7.

It is found that both obtained cathodic and anodic curves exhibit Tafel-type behavior. The addition of the inhibitor increased both cathodic and anodic over potentials, and caused parallel displacement to the more negative and positive values, respectively. From inspection of the values of $i_{\text {corr }}$, it is inferred that the addition of inhibitor decreases the corrosion process of aluminum in $0.5 \mathrm{~N} \mathrm{HCl}$, and the degree of inhibition depends on the inhibitor's concentration. 


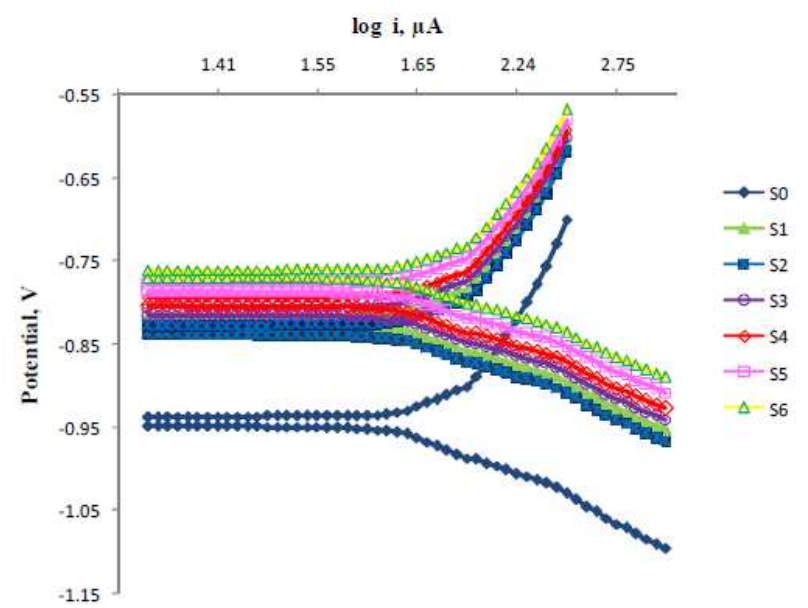

Figure 11. Polarization curves for aluminum dissolution in $0.5 \mathrm{~N} \mathrm{HCl}$, in the presence of different concentrations of $\mathrm{EEPgS}$ at room temperature $(303 \mathrm{~K})$.

Table 7. Effect of EEPgS concentration on the corrosion current density ( $\left.\mathrm{i}_{\text {corr }}\right)$, corrosion potential $\left(E_{\text {corr }}\right)$, Tafel slopes $\left(\beta_{\mathrm{a}}\right.$ and $\left.\beta_{\mathrm{c}}\right)$, degree of surface coverage $(\theta)$, and inhibition efficiency (IE\%) for the corrosion of aluminum in $0.5 \mathrm{~N} \mathrm{HCl}$ at $303 \mathrm{~K}$.

\begin{tabular}{|c|c|c|c|c|c|c|}
\hline Solution & $\begin{array}{c}\text {-Ecorr } \\
\text { (in } \mathrm{mV} \text { ) }\end{array}$ & $\begin{array}{c}\text { icorr } \\
\left(\text { in } \underset{\mu}{\mu} \mathbf{c m}^{-}\right. \\
2\end{array}$ & $\begin{array}{c}\beta_{a} \\
m V \text { dec }^{-1}\end{array}$ & $\begin{array}{c}\text { Bc } \\
\mathrm{mV} \mathrm{dec}\end{array}$ & IE\% & $\boldsymbol{\theta}$ \\
\hline S0 & 945 & 63.1 & 218 & 91 & - & - \\
\hline S1 & 943 & 53.8 & 201 & 106 & 58 & 0.58 \\
\hline S2 & 940 & 52.3 & 203 & 110 & 63.1 & 0.63 \\
\hline S3 & 941 & 50.7 & 204 & 111 & 67.2 & 0.67 \\
\hline S4 & 939 & 46.9 & 206 & 114 & 71.3 & 0.71 \\
\hline S5 & 938 & 43.6 & 210 & 117 & 76.3 & 0.76 \\
\hline S6 & 937 & 39.7 & 212 & 119 & 81.2 & 0.81 \\
\hline
\end{tabular}

It could be derived from this decrease that the rate of anodic dissolution was much retarded, in comparison to that of the cathodic hydrogen evolution. The data obtained also suggest that the inhibitor acts as mixed type inhibitor, because it is enhancing both the cathodic and anodic processes [34-35]. The values of $E_{\text {corr }}$ shift to less negative values with the increase in the inhibitor's concentration. Values of IE\% and $\theta$ are in excellent agreement with the values of weight loss technique.

\section{Scanning Electron Microscopy (SEM) analysis}

The aluminum alloy (AA6063) specimens used to study the corrosion inhibition effect of EEPgS in the acidic environment were also studied with SEM [36]. The SEM photographs for the corroded (with and without inhibitor) coupons are seen in Figs. 12 and 13. The surface morphologies reinforce the inference that the inhibitor protects the decay of metal surface [36-37]. It is clearly seen that the metal surface dipped in $0.5 \mathrm{~N} \mathrm{HCl}$ without inhibitor had more pronounced depth of pitting compared to that containing EEPgS. 


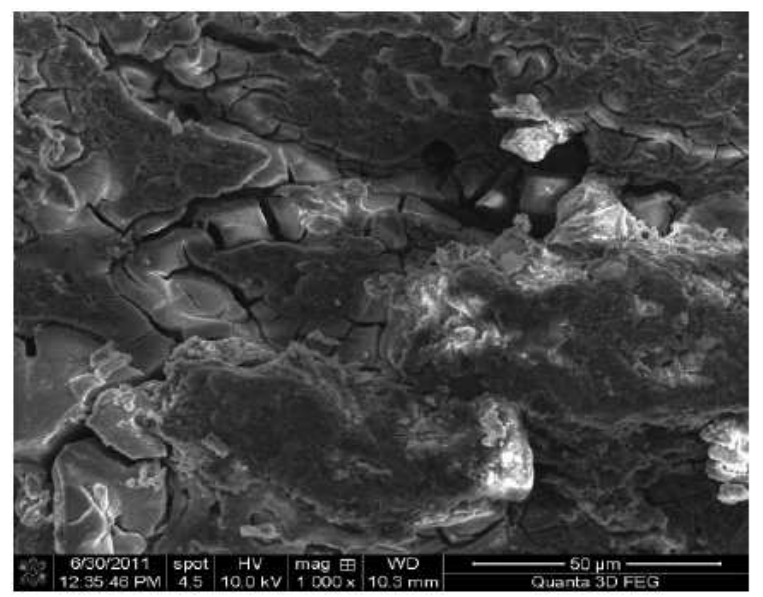

Figure 12. Microstructure of aluminum alloy (AA6063) surface corroded in the presence of $0.5 \mathrm{~N} \mathrm{HCl}$.

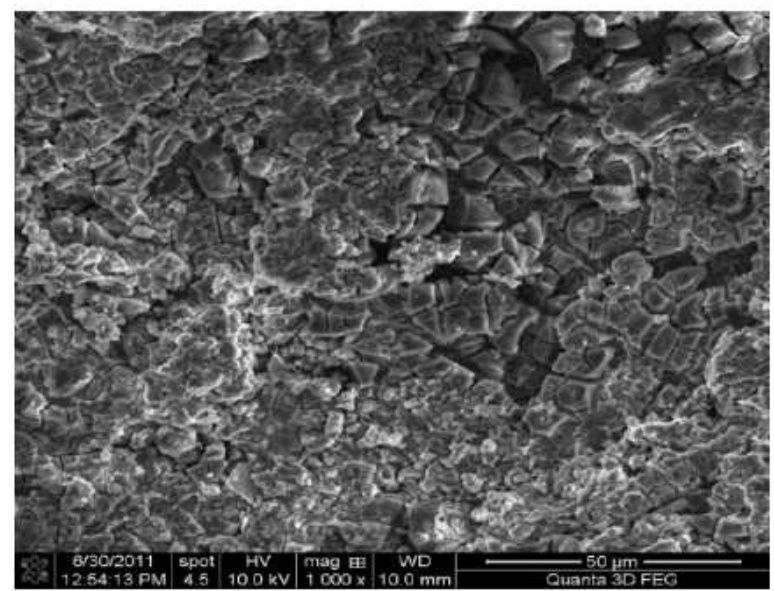

Figure 13. Microstructure of aluminum alloy (AA6063) immersed in $0.5 \mathrm{~N} \mathrm{HCl}$ in the presence of inhibitor solution S6 (1.206 g/L), highest IE\% (85.71\%).

\section{Conclusions}

This work investigated the properties of corrosion inhibition of EEPgS on aluminum in $0.5 \mathrm{~N} \mathrm{HCl}$, using weight loss method. The analysis of the results showed that the inhibition efficiency increased with an increase in the inhibitor concentration, but decreased with an increase in the temperature. Analysis of FTIR results showed that the inhibition mechanism was by adsorption process, through the functional groups present in the extract. The physical adsorption mechanism of the inhibition process was in confirmation with the data obtained, and Langmuir model was best fitted with the results. The calculated activation energy values also confirmed a physical adsorption mechanism. The SEM images of the aluminum sampled shows that the metal was protected in the presence of the extract.

\section{References}

1. Sastri VS. Corrosion inhibitors: principles and applications. United Kingdom: Wiley; 1998. 
2. Sastri VS. Green Corrosion Inhibitors: Theory and Practice. United Kingdom: Wiley; 2012.

3. Ali SK, Saeed MT, Rahman SU. Corros Sci. 2003;45:253.

4. Shorky H, Yuasa M, Sekine I, et al. Corros Sci. 1998;40:2173.

5. Obot IB, Obi-Egbedi NO, Umoren SA. Int J Electrochem Sci. 2009;4:863.

6. Noor EA. J Appl Electrochem. 2009;39:1465.

7. Oluseyi OA, Olugbenga AO, Kolawole OA, et al. J Eng. Appl Sci. 2011;6:10.

8. Umoren SA, Obot IB, Ebenso EE, et al. Desalination. 2009;250:225.

9. Khandelwal R, Sahu S, Arora SK, et al. J Corros Sci Eng. 2010;13:1466.

10. Chauhan R, Garg U, Tak RK. E-J Chem. 2011;8:85.

11. Wilson PG, Brien O, Marcelle M, et al. Am J Bot. 2011;88:2013.

12. Joseph B, Priya RM. Int J Pharm Bio Sci. 2011;2:211.

13. Michel HN, Salib JY, Ishak MS. Pharma Zie. 2002;57:859.

14. Ahmed RS, Yagoub SO. Res J Microbio. 2007;2:845.

15. Nicanor B, Moreno A, Ayala AL, et al. J Food Bio Chem. 2001;25:77.

16. Rajendran S, Jeyasundari J, Usha P, et al. Port Electrochim Acta. 2009;27:153.

17. James AO, Akaranta O. Res J Chem Sci. 2011;1:3.

18. Noor EA. Int J Electrochem Sci. 2007;2:996.

19. Saratha R, Priya SV, Thilagavathy P. E-J Chem. 2009;6:785.

20. Chauchan LR, Gunaskaran G. Corros Sci. 2007;49:1143.

21. Cramer SD, Covino BSJr. In: ASM Handbook. Volume 13A. Corrosion: fundamentals, testing, and protection. US: ASM International; 2003.

22. Fontana MG. In: Corrosion: a compilation. Columbus: The Press of Hollenback; 1957.

23. Fontana MG, Greene ND. Corrosion engineering. New York: McGrawHill; 1967.

24. Bard AJ, Faulkner LR. Electrochemical methods, fundamentals and applications. 2nd ed. New York: John Wiley \& Sons; 2000.

25. Bokris JO'M, Reddy AKN. Modern electrochemistry - electrodics in chemistry, engineering, biology and environment science. 2nd ed. New York: Plenum Publishers; 2000.

26. Kalsi PS. Spectroscopy of organic compounds. 6th ed. New Delhi: New Age Int Publ; 2004.

27. Li S. Monitoring corrosion using vibrational spectroscopic techniques. In: A Tiwari A, Hihara L, Rawlins J, editors. Intelligent coatings for corrosion control. UK: Butterworth-Heinemann; 2015.

28. Jain T, Chowdhary R, Arora P, et al. Bull Electrochem. 2005;21:23.

29. Nahl'e A, Abu-Abdoun I, Abdel-Rahman I, et al. Int J Corros. 2010;460154.

30. Chauhan R, Garg U, Tak RK. E-J Chem. 2011;8:85.

31. Zarrouk A, Warad I, Hammouti B, et al. Int J Electrochem Sci. 2010;5:1516.

32. Ostovari A, Hoseinien SM, Peikari M, et al. Corros Sci. 2009;51:1938.

33. Bouklah M, Hammouti B, Lagrenee M, et al. Corros Sci. 2006;48:2831. 
34. Amin MA. J Appl Electrochem. 2006;36:215.

35. Mansfeld F, Jeanjaquet SL, Kendig MW. Corros Sci. 1986;26:735.

36. Goodhew PJ, Humphreys J, Beanland R. Electron microscopy and analysis. 3rd ed. Oxford: Taylor \& Francis; 2000.

37. Egerton RF. Physical principles of electron microscopy: an introduction to TEM, SEM, and AFM. Berlin: Springer; 2005. 